\title{
La mobilisation des réfugiés palestiniens dans le sillage de la « révolution » syrienne : s'engager sous contrainte
}

The mobilisation of Palestinian refugees in the Syrian "revolution": engaging under duress

\section{Valentina Napolitano}

\section{Q OpenEdition Journals}

Édition électronique

URL : http://journals.openedition.org/conflits/18489

DOI : $10.4000 /$ conflits. 18489

ISSN : $1777-5345$

Éditeur :

CCLS - Centre d'études sur les conflits lilberté et sécurité, L'Harmattan

Édition imprimée

Date de publication : 26 décembre 2012

Pagination : 119-137

ISBN : 978-2-336-00248-4

ISSN : 1157-996X

\section{Référence électronique}

Valentina Napolitano, «La mobilisation des réfugiés palestiniens dans le sillage de la « révolution » syrienne : s'engager sous contrainte », Cultures \& Conflits [En ligne], 87 | Automne 2012, mis en ligne le 26 décembre 2013, consulté le 30 mars 2021. URL : http://journals.openedition.org/conflits/18489 ; DOI : https://doi.org/10.4000/conflits. 18489 


\section{La mobilisation des réfugiés palestiniens dans le sillage de la "révolution» syrienne : s'engager sous contrainte}

\section{Valentina NAPOLITANO}

Doctorante à l'École des hautes études en sciences sociales et boursière à l'Institut Français du Proche Orient (2010-2012), Valentina Napolitano prépare une thèse en Sociologie politique sous la direction de M. Hamit Bozarslan. Ses recherches portent sur les mobilisations politiques et les engagements partisans au sein des camps de réfugiés palestiniens en Syrie.

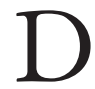

epuis la mi-mars 2011, la Syrie connaît un mouvement de contestation appelant à la fin du régime politique établi, il y a plus de quarante ans, par la famille Assad. Révolte des franges périphériques et largement marginalisées de la société, à ses débuts, la contestation parvient, au fil des mois, à gagner les dimensions d'une véritable mobilisation de masse sur l'ensemble du territoire national ${ }^{1}$. Celle-ci plonge ses racines dans le contexte d'exacerbation des injustices sociales engendré par la politique de libéralisation économique lancée par le président Hafez al-Assad dans les années 1980 et développée par son fils, Bachar, arrivé au pouvoir en 2000. Elle incarne, en outre, les attentes démocratiques et les demandes de liberté d'opinion dans un pays où la peur et la répression ont été au cour du système de coercition qui a assuré la survie du régime.

Parmi les différentes composantes (sociales, ethniques et religieuses) de la société syrienne qui ont rejoint la contestation révolutionnaire, on retrouve une communauté au statut particulier : celle des réfugiés palestiniens qui compte aujourd'hui près d'un demi-million d'individus ${ }^{2}$. Lorsque les mani-

1. Damas et Alep sont les seules villes où, en raison du déploiement massif des forces de sécurité, les manifestations anti-régimes n’ont pas réussi à gagner de façon massive le centre-ville. Cependant, la contestation s'est emparée de certains quartiers circonscrits et des périphéries qui entourent ces deux villes.

2. Selon les statistiques publiées par l'UNRWA (United Nations Relief and Works Agency) en 2010, le nombre de Palestiniens en Syrie est de 496000 individus. Ce chiffre fait toutefois référence exclusivement aux Palestiniens arrivés en Syrie entre 1948 et 1956 sans prendre en 
festations contre le régime ont commencé, les Palestiniens ont essayé de rester à l'écart de la contestation. Cependant, quelques mois plus tard, la peur et l'attentisme du début ont cédé la place à une mobilisation de plus en plus importante en faveur de la « révolution ». Les Palestiniens de Syrie ont ainsi intégré la contestation et cela à partir de leurs propres subjectivités et attentes.

Cet article propose une lecture de l'actuelle contestation syrienne à partir du point de vue palestinien dans une tentative d'apporter de nouveaux éléments de compréhension du terrain révolutionnaire syrien. Si les Palestiniens n'occupent pas une place centrale dans le phénomène contestataire en général, une étude de leur mobilisation nous permettra toutefois d'approfondir notre connaissance des "répertoires d'actions " 3 et des «pratiques de résistance individuelles » ${ }^{4}$ adoptées au cours de la « révolution » syrienne face aux contraintes imposées aux contestataires par la répression du régime.

En raison de leur statut de réfugiés, les Palestiniens sont particulièrement exposés à la répression et disposent d'une gamme limitée de moyens d'action politique. En effet, les Palestiniens de Syrie ont joui d'un statut juridique assez favorable qui leur a permis d'intégrer le tissu social du pays ${ }^{5}$. Cependant, ils ne disposent ni de la nationalité, ni de droits politiques. Ils se trouvent ainsi relégués en marge de la «cité » politique et hors de la «nation » syrienne, et peuvent, à tout moment, devenir des «étrangers ». Leur prise de décision de s'engager au sein de la contestation devient alors plus difficile puisque cela signifie exposer au danger l'ensemble de la communauté. C'est justement à partir de cette condition de dépendance vis-à-vis du pouvoir et d'éternelle précarité que les Palestiniens prennent position au sein de la « révolution».

Dans cet article, nous nous interrogerons sur les motivations qui ont poussé les Palestiniens à rejoindre la « révolution » et sur les attentes dont ils chargent leur mobilisation. Nous essayerons en outre de repérer les formes de mobilisation adoptées par cette communauté et les slogans à travers lesquels elle a exprimé ses propres revendications. En nous intéressant aux mobilisations de la communauté palestinienne dans le sillage de la « révolution » syrienne, nous souhaitons inscrire notre travail dans le débat plus large des mobilisations politiques des populations diasporiques ${ }^{6}$. L'engagement politique des Palestiniens ${ }^{7}$ doit être compris à la lumière du double lien que ces

considération ceux arrivés au cours des vagues d'émigration successives.

3. Tilly C., "Contentious Repertoires in Great Britain, 1758-1834", in Traugott M. (ed.), Repertoires and Cycles of Collective Action, Durham, Duke University Press, 1995, p. 26.

4. Pechu C., «"Laissez parler les objets !" De l'objet des mouvements sociaux aux mouvements sociaux comme objets », in Favre P. (ed.), L'atelier du politiste, Paris, La Découverte, 2007, p. 63.

5. Ce sujet sera traité dans les détails dans le paragraphe suivant.

6. Sur ce sujet voir : Grojean O., La cause kurde, de la Turquie vers l'Europe. Contribution à une sociologie de la transnationalisation des mobilisations, Thèse de doctorat en Sociologie Politique, EHESS, 2008 ; Sokefeld M., "Mobilizing in transnational space: a social movement approach to the formation of Diaspora”, Global Networks, vol. 6, n³, 2006, pp. 256-283. 
derniers entretiennent à la fois avec le pays d'accueil, la Syrie, où ils sont installés depuis longtemps, et le pays d'origine, la Palestine, qui demeure leur référence en termes d'appartenance nationale. C'est en vertu de cette double appartenance que la « révolution » est devenue le théâtre pour l'expression non seulement des quêtes de liberté et dignité du peuple syrien, mais aussi des revendications proprement palestiniennes s'inscrivant dans un contexte national marqué par le mécontentement généralisé des réfugiés vis-à-vis de leur leadership politique.

L'inscription de notre travail au sein des études sur les diasporas permet, en outre, de mener une réflexion autour du rôle que ces populations jouent dans des situations de conflit. Plusieurs études ont montré que les diasporas tentent d'influencer les conflits en œuvre dans leur « bomeland à travers des actions de solidarité, de financement, de lobbying ou d'armement 8 . Cependant, très peu d'études se sont interrogées sur la façon dont les diasporas interagissent et interviennent dans des conflits qui traversent leur pays « hôte ». Le terrain syrien nous permettra donc d'interroger la façon dont la diaspora palestinienne réagit vis-à-vis de la situation de crise provoquée par la « révolution » syrienne et cela en termes de représentations et de mobilisations.

Les informations utilisées dans cette étude proviennent de plusieurs sources. Tout d'abord des observations et des entretiens effectués au cours d'une enquête de terrain, réalisée entre septembre 2010 et mai 2011 au sein du camp palestinien de Yarmouk ${ }^{9}$, à la périphérie de Damas, et portant sur les engagements et les mobilisations politiques des réfugiés palestiniens. Présente sur le terrain au début de la « révolution », entre mars et mai 2011, nous avons pu participer à des conversations informelles autour des manifestations qui commençaient alors à gagner la Syrie. À partir de mai 2011, d'autres informations ont été récoltées à travers des entretiens par téléphone ou internet grâce au logiciel skype. Ces derniers ont été conduits auprès des Palestiniens résidant en Syrie avec lesquels nous avons gardé contact depuis le précédent séjour. Cet article s'appuie principalement sur trois entretiens réalisés auprès des Palestiniens qui ont été impliqués dans la contestation (Hossam, Safaa et Ali) et sur des conversations plutôt informelles qui ont servi à récolter des informations ponctuelles. Enfin, les réseaux sociaux ont été utilisés dans le but de récolter du matériel vidéo, des affiches et des communiqués concernant les

7. Sur l'utilisation du terme « diaspora » en référence aux réfugiés palestiniens voir : Peteet J., "Problematizing a Palestinian Diaspora", International Journal of Middle East Studies, vol. 39, 2007, pp. 627-646 ; Doraï K., «Les Palestiniens : vers l'émergence d'une diaspora de réfugiés ? ", in Sheffer G. (ed.) Les diasporas : 2000 ans d'histoire, Rennes, Presses Universitaires de Rennes, 2005, p. 215.

8. Voir Demmers J., "New wars and Diasporas: suggestions for research and policy", Journal of Peace Conflict E Developpement, vol. 11, 2007.

9. Yarmouk a été créé entre 1954 et 1957 et représente aujourd'hui le plus grand agglomérat palestinien en Syrie avec près de 148500 réfugiés. 
mobilisations organisées dans les camps de réfugiés au cours de la période révolutionnaire.

Cet article sera structuré autour de trois parties. Il s'agira d'abord de présenter les conditions, sociales et politiques, des Palestiniens en Syrie. Cela servira de cadre pour mieux saisir les subjectivités à partir desquelles les Palestiniens apportent leur lecture de la contestation. Par la suite, nous essayerons de montrer quelles sont les différentes formes de mobilisations individuelles et collectives qui ont vu les Palestiniens pour protagonistes. Il s'agira, d'une part, de comprendre comment les mobilisations palestiniennes sont porteuses des mêmes revendications que celles des Syriens et d'autre part, comment celles-ci deviennent une opportunité pour les Palestiniens d'exprimer des attentes qui leur sont propres.

\section{Subjectivités palestiniennes}

\section{Intégrés socialement, mais toujours des étrangers}

La plupart des Palestiniens qui résident actuellement en Syrie 10 sont les descendants des réfugiés arrivés dans le pays suite à la Nakba ${ }^{11}$ de 1948. De nouvelles vagues de déplacement se sont vérifiées en Syrie suite aux conflits qui ont bouleversé la région : notamment après la Guerre des Six jours en 1967, lors des événements de « Septembre noir » en 1970, suite à l'invasion israélienne du sud du Liban en 1982 et la guerre du Golfe en 1990.

Les Palestiniens de Syrie sont souvent cités comme ayant bénéficié de meilleures conditions d'accueil par rapport à d'autres pays hôtes, tel le Liban, où les Palestiniens font l'objet de mesures discriminatoires 12. En Syrie, les Palestiniens ont obtenu les mêmes droits que les citoyens syriens en matière de travail, d'éducation et de santé, selon la loi n 260 adoptée en 1956. L'attribution d'un statut juridique favorable aux Palestiniens s'explique à la fois par la politique nationaliste adoptée par les autorités de l'époque, ainsi que par le fait que les Palestiniens ne dépassent pas $3 \%$ de la population et ne menacent donc pas la stabilité de la Syrie.

Des distinctions entre Palestiniens et Syriens existent tout de même en ce qui concerne la nationalité, puisque les Palestiniens ont gardé leur nationalité, tout en disposant d'un permis de voyage syrien en guise de passeport. D'un

10. La majorité de la population palestinienne se trouve répartie entre douze camps de réfugiés situés à proximité des principales villes syriennes. Actuellement près des trois quarts de la population palestinienne vivent autour de la capitale syrienne en raison des plus nombreuses opportunités de travail.

11. Mot arabe qui signifie « catastrophe ». Ce terme est utilisé depuis les années 1950 pour désigner la formation, en 1948, de l'État d'Israël sur les territoires de la Palestine historique et l'exode de milliers de Palestiniens dans les pays arabes voisins.

12. Au Liban, les Palestiniens sont l'objet d'une série de discriminations notamment en ce qui concerne l'accès au marché du travail, à l'enseignement public et aux services sociaux. 
point de vue politique, ils n'ont pas le droit de voter ou de se porter candidat au sein du Conseil du peuple ${ }^{13}$. Enfin, ils n'ont pas le droit de posséder plus d'un bien immobilier par personne.

Grâce au statut juridique qui leur a été accordé et au niveau d'éducation qu'ils ont atteint, les Palestiniens ont pu effectuer en Syrie une ascension sociale importante en investissant massivement le secteur public et celui des professions libérales. Cette ascension sociale n'a pas toujours été bien vue par les franges les plus démunies de la population syrienne, notamment parmi ceux venants des campagnes et qui se sont urbanisés au cours des années soixante-dix. Ces derniers, contrairement aux Palestiniens qui étaient implantés depuis longtemps dans l'agglomérat urbain de Damas et disposaient d'un capital culturel, avaient moins de chance de trouver un emploi dans la ville ${ }^{14}$. Ils voyaient les Palestiniens comme de véritables concurrents qui auraient même «volé le travail aux enfants du pays » d'après Ali, Palestinien âgé de 56 ans, lorsqu'il décrit le regard porté par les Syriens sur sa famille ${ }^{15}$.

L'intégration sociale dont on entend souvent parler en référence aux Palestiniens de Syrie, demeure, en effet, précarisée par des représentations négatives qui continuent jusqu'à présent d'entourer l'image des Palestiniens dans le pays. "Ibn al-mukhayyam (l'enfant du camp) ne sera jamais comme ibn al-balad (l'enfant du pays) » affirme Diab, habitant du camp de Yarmouk, âgé d'une trentaine d'années, lorsqu'il décrit ce qui différencie le camp de l'environnement syrien ${ }^{16}$. Selon lui, les Palestiniens continueraient à être défavorisés en raison de leur statut de réfugiés. Le stigmate pesant de « l'étranger » 17 semble donc être toujours d'actualité pour cette communauté.

Par ailleurs, en raison de leur appartenance à une nation différente, les Palestiniens sont des « hôtes » sur le territoire syrien et en tant que tels, ils doivent respecter les règles politiques du pays, accepter de rentrer dans ses jeux de manipulation et toujours faire preuve de loyauté envers le régime. C'est donc en partant de cette double condition de précarité, mais aussi de dépendance et de subordination des Palestiniens vis-à-vis de leur pays d'accueil, que nous pourrons mieux comprendre la façon dont cette communauté perçoit la contestation révolutionnaire qui traverse aujourd'hui la Syrie. Mais pour comprendre l'actualité, il nous faut revenir sur l'histoire des relations que les Palestiniens de Syrie ont entretenues avec le régime depuis l'ascension de Hafez al-Assad au pouvoir en 1970. Celles-ci ont forgé en profondeur les subjectivités de la communauté tout entière.

13. Principale institution gouvernementale syrienne.

14. Sayigh, Y., Armed Struggle and the Search for State. The Palestinian National Movement, 1949-1993, Oxford-Washington, Clarendon Press Institute for Palestinian Studies, 1997, p. 45.

15. Entretien effectué à Yarmouk en février 2011.

16. Entretien effectué à Yarmouk en décembre 2010.

17. Goffman E., Stigmate. Les usages sociaux des handicaps, Paris, Minuit, 1975, p. 15. 
La politique de la Syrie à l'encontre des Palestiniens et de leurs organisations politiques a connu des phases très différentes. Elle est passée du soutien des factions, notamment du Fatah au début des années 1960, à la cooptation et au conflit ouvert avec celles-ci au cours des années 1970-1980. Lorsque le président Hafez arrive au pouvoir, sa politique vis-à-vis du Mouvement National palestinien (MNP) se stabilise toutefois autour de deux lignes conductrices. Le président syrien adopte une approche de type "interventionniste ", pour reprendre les mots de Yazid Saygh ${ }^{18}$, dans le sens qu'il vise à intervenir directement sur la vie politique palestinienne à travers des pratiques politiques multiples. Le président prône aussi une politique pragmatique s'inscrivant dans le contexte de la défaite arabe de la guerre de 1967 et dont le but principal serait celui de préserver les intérêts nationaux et contenir les groupes armés palestiniens basés sur le territoire syrien.

Ses rapports avec le MNP deviennent pour Hafez al-Assad un enjeu central de sa politique régionale, il s'en sert notamment comme une carte politique afin d'assurer la défense de ses intérêts au Liban. C'est justement dans le contexte libanais que nous pouvons repérer trois moments historiques qui ont constitué de véritables moments de « ruptures» dans le rapport des Palestiniens avec le régime syrien. Il s'agit de l'intervention syrienne dans la guerre du Liban en 1976, de la guerre de Tripoli en 1983 et de la guerre des camps entre 1985 et 1987. Si d'autres moments historiques ont marqué les relations syro-palestiniennes, tel le massacre de Tell al-Zaatar ${ }^{19}$, ces trois dates sont celles qui correspondent à des vagues de répression massives du régime à l'encontre des Palestiniens de Syrie, qui à plusieurs reprises ont manifesté leur opposition à la politique syrienne.

En 1976, l'intervention de l'armée syrienne pour contrer l'essor du front progressiste, constitué par le Mouvement National libanais (MNL) et l'Organisation de libération de la Palestine (OLP), dans le cadre de la guerre civile, provoque un véritable choc parmi les Palestiniens. En Syrie, les réfugiés manifestent contre cette intervention et en septembre 1976 un commando palestinien effectue une prise d'otages à l'hôtel Samiramis, au centre de Damas. De son côté le régime répond à ce mécontentement par une vague d'arrestations dans les camps de réfugiés. Ali nous raconte qu'en 1976, il était étudiant à l'Université de Damas. Il faisait partie d'un groupe marxiste et lorsque l'armée syrienne envahit le Liban, il participe à l'organisation de mani-

18. Sayigh Y., “The politics of Palestinian exile”, Third World Quarterly, vol. 9, n 1, 1987, p. 41.

19. Le camp de Tell al-Zaatar, situé dans la partie est de Beyrouth, abritait près de 50000 Palestiniens. Durant la guerre civile libanaise les milices chrétiennes décident d'homogénéiser ce secteur de la capitale en éliminant les éléments non-chrétiens. Le camp sera alors assiégé pendant cinquante jours, faisant un millier de victimes. La Syrie sera accusée d'avoir participé au massacre des Palestiniens où au moins d'avoir laissé faire les milices chrétiennes sans intervenir. 
festations en opposition à cette décision. Recherché par les autorités syriennes, il est alors obligé de fuir la Syrie. Il se rend dans les camps palestiniens au Liban mais, un an plus tard, lorsqu'il rentre en Syrie, il est emprisonné pendant huit ans 20 .

Un autre événement clé est représenté par la Bataille de Tripoli qui consolide la scission d'une branche dissidente au sein du Fatah, le Fatah alIntifada 21. Ce dernier est soutenu par la Syrie qui l'accueille sur son territoire et lui donne le feu vert pour qu'il s'approprie l'ensemble des structures civiles et militaires que le Fatah possède dans les camps. À cette époque, les Palestiniens restés loyaux à Yasser Arafat sont arrêtés et le mouvement est officiellement interdit 22 .

Enfin, la guerre des camps, au cours de laquelle la Syrie mobilise le mouvement chiite Amal contre l'OLP, des manifestations de protestation contre la politique syrienne sont à nouveau organisées dans le camp de Yarmouk et réprimés par le régime. Majid Kayyali, écrivain et ex-militant du Fatah, âgé de 58 ans, décrit cette guerre comme "un des événements clé pour comprendre la rage palestinienne contre le régime syrien » 23 . Tout au long du régime d'Hafez al-Assad, les Palestiniens de Syrie ont donc directement expérimenté la manipulation et la coercition du pouvoir.

Avec la succession de Bachar al-Assad, une nouvelle période dans la «politique palestinienne » de la Syrie semble débuter. Sur la scène interne, les Palestiniens profitent du climat d'apaisement suscité par les espoirs de changements dont le nouveau président semble être porteur. Sur la scène régionale, le régime adopte la rhétorique de la «mumana'a» (refus) et, avec l'Iran, le Hezbollah libanais et le Hamas palestinien, il prône la lutte armée contre Israël et contre l'hégémonie américaine au Moyen-Orient. Cette politique semble lui donner une certaine légitimité autant parmi les Syriens que parmi les Palestiniens qui voient d'un bon œil le rapprochement du régime avec le Hamas qui, depuis les années 2000, commence à jouer un rôle politique central en Palestine et dans la diaspora. Cependant, si la rhétorique de la « résistance » parvient à mobiliser les faveurs d'une partie des réfugiés, ces derniers restent toujours conscients des visées instrumentales du régime et la révolution de 2011 deviendra le moment propice pour dénoncer la politique syrienne à l'encontre des Palestiniens.

20. Entretien effectué à Yarmouk en février 2011.

21. Faction issue du Fatah suite à la scission du leader Abou Mousa, en 1993.

22. Les partisans du Fatah continuent d'organiser des activités en Syrie, mais le mouvement ne dispose pas d'une base dans le pays.

23. Entretien effectué sur skype en février 2012. 


\section{Mobilisations et pratiques de résistance}

\section{Le dilemme d'être réfugiés}

En février 2011, le régime de Hosni Moubarak chute. Dans les rues du camp de Yarmouk l'enthousiasme éclate et l'ambiance se fait vibrante. On peut entendre les cris de joie venant des maisons, voir les gens s'échanger de timides félicitations et, dans les boutiques du grand marché du camp ${ }^{24}$, les écrans de télévision restent syntonisés sur les informations. Un nouvel espoir gagne la région, et pour les Palestiniens de Syrie qui lisent l'événement à la lumière de leur condition, une volonté de changement fait surface. «Je pense que les intifada tunisienne et égyptienne vont redonner une nouvelle âme à la cause palestinienne - affirme Ali - de cet optimisme naîtra une nouvelle génération de Palestiniens ! 25

Cependant, lorsqu'à la mi-mars 2011, l'écho des mouvements révolutionnaires se propage en Syrie et que les premières manifestations anti-régime apparaissent dans le sud du pays, à Deraa', les réactions des Palestiniens sont mitigées par la peur. Les Palestiniens craignent non seulement la réaction du régime, mais aussi d'être les premiers à payer les frais d'une déstabilisation syrienne. À plusieurs reprises dans l'histoire, les Palestiniens ont, en effet, été les victimes des crises qui ont traversé les pays arabes d'accueil. En Jordanie en 1970, au Liban en 1975, au Koweït en 1990 et plus récemment en Irak en 2003, les Palestiniens sont devenus les «ennemis de l'intérieur » voire les " ennemis de la nation ». Lorsque les autorités syriennes ont essayé, au début de la contestation, d'identifier des boucs émissaires extérieurs à la Syrie comme responsables d'un « complot » orchestré contre lui, les Palestiniens du camp de Latakieh ont été les premières cibles de cette stratégie. Buthaina Shaaban, conseiller du président, les aurait même accusés d'avoir commencé la "fitna ( sédition) en attaquant des commerçants du centre-ville ${ }^{26}$. Cet événement a ainsi réveillé une "mémoire collective " encore vivante parmi les réfugiés et qui les a poussés à adopter une attitude très prudente face aux événements. Cette posture est semblable à celle adoptée par les communautés minoritaires syriennes : les minorités confessionnelles qui craignent qu'un changement de régime puisse affecter leur liberté de culte, et la minorité ethnique kurde qui, en raison de ses mobilisations passées contre le régime et de ses visées séparatistes, craint que le régime puisse l'identifier comme la responsable de la contestation. Les craintes de ces communautés minoritaires sont d'ailleurs alimentées par le régime qui, à travers sa propagande visant à fomenter le spectre de la guerre civile, essaye de taire le soulèvement.

24. Le camp de Yarmouk a connu, à partir des années 1980, une expansion massive des activités commerciales.

25. Entretien effectué en février 2011.

26. Voir l'article de presse : «Les Palestiniens ont commencé la fitna à Latakieh », Al-Watan, en arabe, 27 mars 2011. 
Le manque de prise de parole des Palestiniens peut être expliqué aussi par d'autres raisons. Une partie des réfugiés considère, en effet, avoir bénéficié de conditions de vie bien meilleures en Syrie que dans d'autres pays arabes. Pour cette raison, ces Palestiniens ressentent le devoir de rester loyaux envers le pays d'accueil. D'autres, au contraire, se considèrent comme des «étrangers » qui ne doivent pas se mêler des affaires internes au pays ${ }^{27}$. Ceux qui militent au sein d'une organisation politique palestinienne, quant à eux, sont obligés de se tenir à l'écart de la contestation, faute de quoi ils mettraient en danger l'organisation d'appartenance. Enfin, l'attitude attentiste des Palestiniens visà-vis de la révolution peut aussi s'expliquer par le fait que n'ayant pas de droits politiques, les réfugiés palestiniens partagent dans leur grande majorité les aspirations de liberté et de démocratie des Syriens, mais sont conscients que dans la future démocratie ils n'auront ni place, ni pouvoir d'influence. Leur engagement dans la « révolution » est alors freiné d'autant que la puissante répression, mise en place par le pouvoir, rend le coût de la participation très élevé.

Les calculs de la communauté palestinienne dans le cadre du nouveau contexte politique inauguré par le soulèvement syrien doivent être mis en lien avec la position délicate occupée par cette communauté de réfugiés. Résidents en Syrie depuis plusieurs générations, les Palestiniens se sentent profondément concernés au niveau émotionnel par le mouvement contestataire ainsi que par sa répression. Cependant, ils sont pris au piège par leurs peurs et par leur dépendance vis-à-vis du pouvoir auquel est lié leur futur dans le pays. Cette condition retarde donc leur engagement au sein de la mobilisation syrienne

\section{Pratiques de résistance individuelles: se mobiliser sans être stigmatisés}

Au fil des mois, la contestation populaire syrienne se poursuit et mobilise un nombre de plus en plus important de personnes. Dans ce contexte, on voit alors émerger les premières mobilisations palestiniennes. Au lieu d'organiser des manifestations dans les rues, répertoire d'action principal des Syriens, les Palestiniens optent d'abord pour un engagement de type individuel. Hossam, professeur universitaire sur la quarantaine, et Safa, employée dans le secteur privé, âgée de 35 ans, nous ont raconté comment ils ont commencé à prendre part à des activités en soutien de la « révolution " 28 . Hossam, a commencé en organisant des activités informatives parmi ses étudiants, Safaa en assistant à des réunions entre Palestiniens et Syriens dans « l'objectif d'échanger des opinions et de construire une conscience autour des événements en œuvre ». Tous deux affirment avoir participé à des manifestations anti-régime en dehors du camp de Yarmouk où ils habitent, notamment dans le proche quar-

27. Ces observations sont issues des conversations informelles auxquelles nous avons participé dans le camp de Yarmouk au début de la contestation syrienne.

28. Ces entretiens ont été effectués sur skype en novembre 2011. 
tier damascène de Midan où se déroulent la plupart des manifestations de la capitale. Par ailleurs, Hossam et Safaa commencent tous les deux à prendre la parole sur les réseaux sociaux.

Ces formes d'engagement individuel au sein de la contestation permettent aux Palestiniens d'embrasser la lutte anti-régime menée par le peuple syrien, tout en évitant que l'ensemble de la communauté puisse être stigmatisé et identifié par le pouvoir avec l'" ennemi intérieur ». Elles constituent donc des formes de contournement des contraintes auxquelles sont exposés les Palestiniens.

Mais quelles sont les motivations qui poussent Hossam et Safaa dans leur décision de s'impliquer au sein de la contestation ? Est-ce que leurs attentes sont différentes de celles des Syriens ? Lorsque nous leur avons demandé pourquoi ils avaient décidé de se mobiliser, plusieurs facteurs ont émergé. "Professeur universitaire qui tente d'enseigner à ses étudiants le regard critique sur le monde et qui œuvre pour le changement, j'étais obligé de donner l'exemple et d'accompagner mes enseignements par des actions concrètes ", affirme Hossam. « À cela il faut ajouter que je suis Palestinien, mais que j'éprouve un sens d'appartenance très fort envers la Syrie. Les Syriens m'ont beaucoup donné et moi aussi, je leur ai donné beaucoup. Nous les Palestiniens, nous faisons partie intégrante du tissu social et culturel syrien et c'est donc un devoir pour nous de prendre position aux côtés de la révolution avec tous les moyens possibles. Puis, qui sait... peut être que le chemin vers la libération de la Palestine doit commencer avec la chute du régime en Syrie !! » Le vécu syrien d'Hossam ainsi que sa responsabilité morale sont deux arguments qui expliquent son engagement politique, qui se fait toujours à travers un prisme d'interprétation palestinien, puisque finalement le but de la contestation serait aussi celui de contribuer à l'avancement de la cause palestinienne. Le thème de l'appartenance au pays d'accueil et de la responsabilité vis-à-vis de celui-ci sont deux sujets évoqués aussi par Safaa : «quand j'ai pensé à m’engager, je n'ai pas pensé au fait que je suis palestinienne. J'ai juste pensé en tant qu'être humain puisqu'en Syrie l'humanité est sur le point d'être anéantie. En outre, je suis née en Syrie et je ressens un sentiment d'appartenance envers ce pays et son peuple, comment je pourrais donc me dissocier de ce qui se passe seulement parce que je suis Palestinienne... bien au contraire, c'est en raison de ma nationalité que je me sens encore plus concernée par les injustices sociales et politiques. » En tant que Palestiniens, Hossam et Safaa mettent donc en avant leur identité lorsqu'ils motivent leur engagement contestataire, mais ils affirment aussi leur appartenance au pays d'accueil où ils sont nés et où ils ont grandi, ce qui fait de leur engagement un véritable devoir. 
Si les mobilisations palestiniennes contre le régime syrien demeurent principalement de type individuel, nous remarquons néanmoins qu'au fil du temps la coordination des Palestiniens au sein du mouvement s'amplifie. Dans les camps, les Palestiniens créent des « comités d'organisation » (lijan al-tansiq) à l'instar de ceux formés dans les villes syriennes. Ceux-ci ont pour but d'échanger des informations avec les autres comités syriens et éventuellement de leur fournir de l'aide le moment venu. C'est le cas du camp de Homs où le comité du camp s'est activé pour fournir de l'aide médicale dans un quartier de la ville ravagé par l'intervention de l'armée syrienne. Ces comités ont, en outre, ouvert des pages sur les réseaux sociaux dont ils se servent pour l'affichage de vidéos des cortèges organisés dans les camps, pour diffuser le nom des Palestiniens victimes de la répression du régime et des listes, connues comme les « listes de la honte », dénonçant les Palestiniens qui collaborent avec les services de sécurité syriens.

Ce passage d'un engagement individuel vers une mobilisation de type collectif est devenu de plus en plus évident au cours des derniers mois. À partir de janvier 2012, nous remarquons la multiplication des manifestations dans les rues du camp de Yarmouk et du proche quartier du Tadamon, issu de l'expansion urbaine du camp et habité à la fois par des Palestiniens et par des Syriens. Au cours de ces mobilisations, le slogan le plus scandé par les protestataires est : «Les Palestiniens et les Syriens sont un seul peuple! », ce qui prouve la solidarité des réfugiés avec le mouvement révolutionnaire. Cette solidarité est attestée aussi par des affiches portées par les manifestants sur lesquelles on peut lire «Aux déplacés syriens, les maisons des réfugiés palestiniens sont ouvertes pour vous accueillir! ", slogan faisant référence à l'accueil que les Palestiniens ont reçu en Syrie dans le passé.

L'entrée des Palestiniens, en tant que collectivité, au sein de la contestation semble toutefois intervenir comme résultat de l'instrumentalisation de ceux-ci par le régime en deux circonstances : la commémoration de la Nakba, le 15 mai, et celle de la Naksa 29, le 5 juin. Comme chaque année, ces événements sont l'occasion d'organiser des activités visant à préserver la mémoire collective palestinienne. Mais en 2011, les jours de commémoration ont pris une tournure différente.

Une « Marche du retour » (Masirat al-'Awda) a été organisée à l'occasion des 63 années du début de la $N a k b a$ dans la région frontalière avec les hauteurs du Golan, région occupée par l'armée israélienne depuis 1967. Ce cortège,

29. Terme qui en arabe signifie « rechute » et indique la victoire d'Israël dans la guerre de Juin 1967. 
organisé en coordination avec les Palestiniens du Liban, de Jordanie et des Territoires occupés qui eux aussi ont manifesté dans les zones à proximité d'Israël, s'est achevé par la mort tragique de trois jeunes Palestiniens sous les tirs de l'armée israélienne. Le lendemain, des funérailles impressionnantes sont organisées dans le camp de Yarmouk d'où provenaient ces jeunes. Des milliers de personnes sont descendues dans les rues du camp pour accueillir les dépouilles « des martyrs du retour ». Le cortège funèbre s'est alors transformé en manifestation de masse, la plus importante, semble-t-il, depuis les années 1980.

Cette mobilisation, qui pourrait paraître anodine, a acquis un sens tout à fait particulier dans le contexte de la « révolution ». La « Marche du retour », en effet, n'était pas une manifestation spontanée, bien au contraire, elle était encouragée par le régime syrien et même organisée par ce dernier à travers son affilié palestinien, le FPLP-CG 30, chargé d'organiser le départ des bus conduisant les Palestiniens aux frontières, même si les manifestants n'appartenaient pas à cette organisation. Pour la première fois, le régime syrien accordait à des Palestiniens l'autorisation de se rendre dans cette zone sous occupation militaire israélienne et qui ne peut être visitée qu'avec l'autorisation des autorités syriennes. En orchestrant cette manifestation, la Syrie risquait pour la première fois de mettre en péril l'accord de cessez-le-feu avec Israël qu'elle avait scrupuleusement respecté depuis 1974. En faisant cela, elle visait à envoyer un double message : à l'extérieur (à Israël et aux États-Unis), le but était de montrer le rôle de garant de la stabilité que le pays joue dans la région, et à l'intérieur, le régime cherchait à rappeler à sa population que le véritable ennemi restait Israël et non le régime qui lui, au contraire, a toujours défendu la cause palestinienne.

Dans cette période de bouleversement, un deuxième événement vient marquer les Palestiniens de Syrie. Il s'agit de la commémoration de la Naksa, le 5 juin 2011, pendant laquelle l'expérience du Golan est répétée, mais s'accompagne, cette fois, par un bilan de victimes beaucoup plus lourd : 23 jeunes perdent la vie ${ }^{31}$. Le lendemain, des funérailles sont encore une fois organisées dans le camp de Yarmouk, d'où provenaient 9 des 23 jeunes morts. Cette fois, les slogans scandés par le cortège changent. Si auparavant ils invoquaient la libération de la Palestine ou proclamaient «Le peuple veut la chute d'Israël », ils expriment à cette occasion la rage des jeunes contre le FPLP-CG, accusé de les avoir instrumentalisés pour servir les intérêts du régime. Ils accusent

30. Acronyme de Front Populaire de Libération de la Palestine - Commandement Général. Cette organisation a été créée, en 1968, suite à la scission du FPLP d'un groupe guidé par Ahmad Jibril, ex-militaire de l'armée syrienne. Le FPLP-CG devient le principal allié palestinien du régime syrien puisqu'à travers lui il peut alimenter les divisions dans le champ politique palestinien et contrer la politique de Yasser Arafat, à laquelle Jibril s'était opposé depuis la formation du Fatah en 1959.

31. Information obtenue par un habitant du camp à travers un entretien téléphonique en mai 2011. 
l'Armée de Libération palestinienne (instance contrôlée par l'armée syrienne) et le président Bachar al-Assad de n'avoir opposé aucune résistance contre l'ingérence de l'armée israélienne sur son territoire national : «Un, deux, trois tu étais où Bachar ? », chantaient les manifestants. Les slogans acquièrent enfin une intonation clairement anti-régime : « Le peuple veut la chute du profiteur !» Ce cortège devient donc une occasion pour dénoncer l'instrumentalisation des Palestiniens et de leur cause par le régime syrien.

Si la manipulation du régime provoque l'entrée des Palestiniens au sein de la contestation, la répression qu'il met en place au sein des camps et des quartiers urbains dans lesquels ces derniers sont intégrés constitue un facteur déterminant de l'insertion des réfugiés au sein de la «révolution ». Dans les différents camps, à Dara'a, Homs et Latakieh, Alep, Damas, les Palestiniens se sont trouvés catapultés dans la contestation en raison des interventions de l'armée syrienne. À Latakieh par exemple, le camp palestinien d'Al-Ramle alFilastiniyyi 32 a été massivement bombardé en août 2011. Le camp intègre le plus grand quartier sunnite de la ville. Ce camp a été, à plusieurs reprises, le lieu de déploiement pour des manifestations anti-régime dans lesquelles étaient impliqués Syriens et Palestiniens. Ces derniers partagent la même condition de marginalisation économique et le même sentiment d'être défavorisés face à l'emprise de la communauté alawite ${ }^{33}$, très nombreuse à Latakieh, non seulement sur les instances de pouvoir, mais aussi sur les principales entreprises et sources de subsistance locales. En affirmant que des armes sont cachées dans le camp, l'armée syrienne se livre ainsi à une puissante intervention militaire. Le bilan est de plusieurs victimes parmi les Palestiniens, des milliers d'autres déplacés et la destruction d'une partie du camp. Cet épisode sera d'ailleurs fortement condamné par l'UNRWA et par l'OLP.

Le camp palestinien d'al-'Aidin ${ }^{34}$, à Homs, et le camp de Deraa' ${ }^{35}$, ont été aussi durement touchés par les interventions militaires et les sièges que le régime a imposé, à plusieurs reprises, à ces deux villes. Le camp de Yarmouk a également expérimenté la répression du régime durant cette période. Plusieurs militaires de l'Armée de Libération palestinienne ont été assassinés, ce qui est arrivé aussi dans le camp de Neirab ${ }^{36}$, près d'Alep. Ces derniers auraient refusé de rejoindre l'armée syrienne dans sa tentative d'étouffer le soulève-

32. Construit en 1955 le camp d'Al-Ramle al-Filastiniyyi héberge plus de 10000 Palestiniens selon les statistiques publiées par l'UNRWA en 2010.

33. Les Alaouites constituent près de $10 \%$ de la population syrienne. Depuis l'ascension de Hafez al-Assad au pouvoir cette communauté confessionnelle, de laquelle est issu le président, a été mobilisée massivement pour occuper des postes clés au sein des institutions publiques et militaires.

34. Construit en 1949, ce camp héberge près de 22000 Palestiniens, selon les statistiques publiées par l'UNRWA en 2010.

35. Construit entre 1950-1951, ce camp héberge près de 10500 Palestiniens, selon les statistiques publiées par l'UNRWA en 2010.

36. Construit entre 1948-1950, ce camp héberge près de 20500 Palestiniens, selon les statistiques publiées par l'UNRWA en 2010. 
ment. Toujours à Yarmouk, plusieurs partisans du Fatah, organisation officiellement interdite en Syrie, ont été arrêtés puisqu'ils seraient accusés d'avoir organisé des manifestations au sein du camp. Par ailleurs, des explosions de voitures, dont les responsables demeurent inconnus, ont eu lieu dans le camp et ont fait plusieurs victimes. Dans le camp de Yarmouk la situation a rapidement basculée, en juillet 2012, suite à une manifestation anti-régime au cours de laquelle dix jeunes palestiniens ont perdu la vie sous les tirs des services de sécurité syriens. Cet épisode a été suivi de funérailles publiques qui ont mobilisé des milliers de Palestiniens et Syriens. Le lendemain, les quartiers du sud de la ville Damas, où se trouve le camp, ont été investis par une opération armée qui prouve que désormais la capitale damascène, elle aussi, n'est plus à l'écart de la violence. Toujours dans l'agglomérat damascène, à Baraze, l'intellectuel palestinien Salame Keleh a été arrêté, puis torturé par les services de sécurité et expulsé en Jordanie ${ }^{37}$. Cet épisode prouve que le danger d'expulsion des Palestiniens, notamment ceux qui ne possèdent pas de documents de voyage syriens, demeure toujours une arme dont le régime peut se servir contre les réfugiés palestiniens.

\section{La contestation dans la contestation : dynamiques de changement palesti- niennes}

\section{Les réfugiés remettent en cause leur leadership politique}

Les deux mobilisations proprement palestiniennes qui ont été organisées à l'occasion de la commémoration de la Nakba et de la Naksa, sont non seulement le fruit d'une orchestration du régime, mais elles sont aussi l'expression d'une volonté des Palestiniens de profiter du climat de bouillonnement politique qui traverse le pays pour porter l'attention sur des questions les concernant de plus près, comme la question du «droit au retour » dans leur terre d'origine et leur réintégration dans la lutte nationale palestinienne.

Après la "Marche du retour ", le camp de Yarmouk devient le siège de débats et d'échanges animés surtout par un groupe de jeunes (entre 20 et 35 ans) qui se disent indépendants et qui érigent pendant une semaine une tente au milieu de la rue principale du camp afin de discuter sur la façon de relancer la mobilisation des réfugiés au sein du combat national.

Ces mobilisations ont, en outre, fourni l'opportunité aux réfugiés d'exprimer le mécontentement qu'ils ressentent envers leur classe politique qui les a marginalisés depuis la signature des accords d'Oslo ${ }^{38}$, en 1993. Pendant les

37. Voir l'article de presse "Salame Keleh : la révolution syrienne vaincra et al-Assad tombera », Al-Jazeera, en arabe, 16 mai 2012.

38. Suite à la signature des accords de paix, les principales factions palestiniennes orientent leur action vers les territoires occupés au dépens des camps de réfugiés qui avaient joué un rôle politique central au cours des années 1960-1970. 
cortèges organisés dans la région du Golan, les participants, pour la plupart de jeunes entre 20 et 35 ans, appellent à ne pas afficher les drapeaux des factions, mais seulement des drapeaux palestiniens. Au cours des funérailles des «martyrs du retour » ils crient : «Le peuple veut la chute des factions ! » et s'opposent à une récupération des mobilisations par les formations politiques palestiniennes. Lorsque Maher al-Taher, leader du FPLP 39 en Syrie, se rend dans le cimetière de Yarmouk pour rendre hommage aux jeunes tombés dans les deux cortèges du Golan, il doit faire face à une pluie de pierres jetées par le public. Ahmad Jibril, leader du FPLP-CG, sera accusé d'avoir envoyé les jeunes Palestiniens se faire tuer dans le Golan et le siège de son organisation à Yarmouk sera attaqué et brûlé en partie ${ }^{40}$. Des affrontements entre les gardes de Jibril et les manifestants ont en outre provoqué la mort de trois jeunes. Lorsque le Hamas et le Fatah tentent de faire passer les «martyrs du retour » comme des partisans de leurs organisations, les jeunes de Yarmouk collent des affiches dans le camp démentant cette information. Les factions ont en outre été durement critiquées en raison du silence qu'elles ont gardé face aux multiples attaques que le régime a perpétré à l'encontre des Palestiniens.

Ces événements qui viennent altérer la situation d'apparent immobilisme dans laquelle se trouvait au départ le camp de Yarmouk sont la preuve évidente de l'écart qui existe entre la classe politique palestinienne et une génération de jeunes réfugiés qui ont grandi dans les camps, et qui ont connu le rêve de la libération seulement à travers les récits de leurs parents. C’est dans le contexte de prise de parole engendré par le phénomène révolutionnaire syrien que les Palestiniens eux-mêmes ont trouvé une voix pour exprimer leurs propres revendications.

Selon Hossam, le soulèvement syrien serait un événement qui a fortement touché la nouvelle génération ayant grandi dans le climat de désespoir engendré par le délaissement du camp par les factions historiques, tels le Fatah et les factions de la gauche, et par le manque d'un véritable projet national. Une analyse des profils sociologiques et des carrières militantes des trois Palestiniens interviewés se révèle intéressante afin de dresser des hypothèses sur les dynamiques générationnelles qui ont animé ces mobilisations. Safaa et Hossam, 35 et 39 ans, sont deux jeunes instruits, l'un travaille dans une entreprise privée, l'autre est professeur d'université. Tous deux ont grandi dans le camp de Yarmouk, mais aucun d'entre eux ne s'est engagé auprès d'une formation politique. Safaa a été active dans le domaine artistique, alors que Hossam a été actif dans celui des droits de l'homme, notamment dans la période d'ouverture politique, connue comme le « Printemps de Damas », entre 2000 et 2001, qui suit l'ascension de Bachar al-Assad au pouvoir. À leur image, nombreux sont

39. Acronyme de Front Populaire de Libération de la Palestine, organisation créée en 1967 par George Habache.

40. Information obtenue par un habitant du camp à travers un entretien téléphonique, en mai 2011. 
les jeunes qui partagent cette même condition de détachement vis-à-vis du milieu politique officiel. Un deuxième profil militant est celui d'Ali, moins engagé sur le terrain de la contestation mais qui partage la même envie de changement portée par les Syriens et par les Palestiniens. Ali est un intellectuel, âgé de 56 ans, ancien membre du FPLP. Dans sa jeunesse il milite dans une organisation marxiste. Il est emprisonné à plusieurs reprises sous le régime d'Hafez al-Assad. À la fin des années 1980, il s'éloigne du FPLP, même s'il continue à travailler avec ce dernier dans l'organisation d'activités culturelles. Comme Ali, nombreux sont les Palestiniens qui, après les accords d'Oslo, ont décidé, ou ont été obligés, faute de financement, de quitter les organisations politiques dans lesquelles ils avaient milité. Ces derniers sont aussi ceux qui, pendant leur carrière militante au sein du MNP, ont enduré les jeux de manipulation et de répression du pouvoir syrien. En ce sens, le mouvement révolutionnaire représente non seulement une cause à soutenir mais aussi une cause à laquelle ils vont s'identifier. Les Palestiniens comme Ali sont ceux qui, au cours de leur carrière militante, ont déjà amorcé une « rupture » avec le milieu politique d'appartenance. Alors que la génération des jeunes profite de la contestation en ouvre pour affirmer sa propre « rupture » vis-àvis des factions qui, à leur avis, incarnent le déclin de la lutte nationale et sont monopolisées par des «vieux » qui manquent de projet politique dans lequel se reconnaître ${ }^{41}$. C'est en ce sens qu'on assiste alors à la mise en place d'une véritable « contestation dans la contestation».

\section{Quelle place pour le Hamas en Syrie?}

La contestation syrienne vient ébranler la vie politique des camps palestiniens non seulement "par le bas ", provoquant la mobilisation des réfugiés, mais aussi "par le haut ", remettant en cause le rapport de certains acteurs politiques avec le pouvoir. Parmi les factions palestiniennes, le Hamas, a été la plus touchée par la contestation.

Il faut d'abord souligner que le mouvement palestinien, présent en Syrie depuis 1993, a joué à partir des années 2000 un rôle hégémonique dans la vie des camps de réfugiés et notamment dans celle de Yarmouk. Cet acteur parvient à gagner une certaine popularité auprès des réfugiés en correspondance avec l'éclatement de la seconde Intifada 42 au sein des Territoires occupés. Relançant les actions armées contre Isräl, le mouvement s'affiche alors comme le seul acteur capable de poursuivre la « résistance ». Grâce aux importantes sources financières dont il dispose et grâce aussi à la marge d'action qui lui est accordée par le pouvoir syrien, le mouvement fonde des structures

41. Ces observations sont issues d'un entretien effectué à Damas, avec Hazem, Palestinien âgé de trente ans, partisan du Fatah et fondateur d'une association pour jeunes.

42. Soulèvement populaire qui éclate dans les territoires occupés en septembre 2000 au lendemain de la visite controversée d'Ariel Sharon sur l'esplanade des mosquées à Jérusalem. Le nom seconde Intifada ou Intifada al-Aqsa, du nom de la mosquée de Jérusalem, fait référence au soulèvement de 1987. 
sociales et organise de nombreuses activités de mobilisation au sein des camps. L'œuvre sociale devient ainsi un des moyens principaux pour gagner le soutien d'une vaste portion de la société palestinienne en Syrie.

Or depuis l'éveil de la contestation syrienne, le rôle du Hamas en Syrie semble bousculé. Au début de la contestation, le Hamas a essayé de garder une attitude neutre. Lorsqu'il est sollicité par le pouvoir pour exprimer une position de soutien au régime, il évite de se prononcer sous prétexte qu'il s'agit d'une affaire interne à la Syrie. Cette posture se justifie à la fois par la volonté du mouvement de prendre du temps pour mesurer l'ampleur des événements, mais aussi dans une volonté de ne pas exposer la communauté palestinienne locale à la vengeance du régime en cas de conflit avec ce dernier. Cette attitude est aussi adoptée par les autres factions palestiniennes présentes sur le territoire syrien, notamment les factions de la gauche, tel le FPLP et le FDLP 43. Ces dernières préfèrent ne pas se prononcer sur l'actualité, alors que le FPLPCG prône le même discours que celui du régime, selon lequel la Syrie serait victime d'un complot international, et participe directement à la répression des manifestations dans les camps.

Avec la poursuite de la contestation, les factions de la gauche, en raison de leur faiblesse et de l'absence d'une alternative à la base syrienne, continuent à garder le silence, alors que le Hamas semble changer de position. Les déclarations des leaders du mouvement en soutien au peuple syrien commencent à remplacer le silence initial. La première déclaration officielle concernant les événements arrive à la fin de décembre 2011. Suite aux explosions qui ont lieu dans le quartier de Midan, à Damas, le Hamas publie sur son site officiel un communiqué dans lequel il déclare que le «sang syrien est cher » et plaide pour « une sortie politique de la crise ».

Mais le véritable basculement du Hamas en faveur de la « révolution » arrive seulement à la fin du mois de février 2012 suite au discours tenu par Ismaël Haniyeh, Premier ministre du gouvernement de Gaza, dans la mosquée Al-Azhar du Caire. «Je salue le peuple syrien, ce peuple héroïque qui dans son pays a ouvert le chemin pour la liberté, la démocratie et la réforme », affirme-t-il alors ${ }^{44}$. Cette prise de position intervient dans un contexte marqué par la montée de la violence et l'isolement croissant de la Syrie sur la scène internationale. Elle est, en outre, encouragée par la victoire, au cours des élections législatives égyptiennes, en janvier 2012, des Frères Musulmans, organisation de laquelle le mouvement palestinien est issu. En mesurant les changements en ouvre dans la région, le Hamas décide alors de rejoindre les positions de l'organisation mère en faveur de la « révolution » syrienne.

43. Acronyme de Front Démocratique de libération de la Palestine fondé en 1969 par Nayf Hawatme.

44. Voir l'article de presse "Le Hamas abandonne al-Assad et soutient l'intifada syrienne », $A l$ Quds al-Arabi, en arabe, 24 février 2012. 
Le «front du refus » qui unissait le mouvement palestinien à la Syrie, l'Iran et le Hezbollah libanais semble donc avoir été brisé. Les leaders du Hamas auraient quitté la Syrie comme le confirment les déclarations faites par Mousa Abou Marzouq, porte-parole du Bureau politique, selon lequel le Hamas ne reviendra pas dans le pays en raison « des actions monstrueuses que le régime d'al-Assad est en train d'accomplir à l'encontre de ses opposants» 45 .

La remise en question de l'alliance avec la Syrie amène le Hamas à redéfinir sa stratégie régionale. C'est pour cela que le mouvement décide de parvenir à une réconciliation avec le Fatah ${ }^{46}$, en mai 2011, et de se rapprocher de l’Égypte, du Qatar et de la Jordanie, qui pourraient fournir une alternative à l'allié syrien.

À l'échelle locale du camp de Yarmouk, la fin de l'alliance avec la Syrie et le départ des leaders du Hamas du pays a eu pour résultat un affaiblissement de la mobilisation et des activités sociales organisées par le mouvement. Par ailleurs, le Hamas n'a pas été épargné par la vague de contestation soulevée par les réfugiés contre les factions palestiniennes. Si dans les dernières années ce mouvement avait gagné un large soutien parmi les Palestiniens, il semble toutefois avoir perdu du terrain et ne plus incarner les espoirs de changement portés par les réfugiés. Après avoir participé aux élections législatives en 2006, intégrant ainsi le système politique issu des accords d'Oslo, et après avoir pris le pouvoir dans la bande de Gaza en 2007, le Hamas n'est plus associé à l'image du mouvement porteur de la « résistance », mais il est considéré, au même titre que le Fatah, comme un mouvement principalement intéressé par la prise de pouvoir. Les réfugiés qui avaient vu dans le Hamas le seul acteur capable d'assurer le renouveau du combat national et l'intégration des réfugiés sur la scène politique, éprouvent aujourd'hui un sentiment de déception à son égard.

L'engagement des Palestiniens au sein de la « révolution » syrienne montre bien toute la difficulté d'une communauté diasporique à intervenir au sein de la vie politique de son pays d'accueil. Si, d'une part, les Palestiniens éprouvent un sentiment de solidarité envers les Syriens et ressentent l'envie de prendre part à la mobilisation contestataire, ils sont, de l'autre, freinés par la contrainte que leur impose leur statut de réfugiés et donc d' «étrangers » dans ce pays. Cette communauté essaye toutefois d'utiliser des registres d'action multiples afin de contourner ces contraintes et de se poser en acteur politique. Dans un premier temps, elle adopte des répertoires moins visibles dans l'espace public, elle se charge de l'information et de la fourniture d'aide médicale,

45. Voir l'article de presse « Mousa Abou Marzouk : les leaders du Hamas ont quitté la Syrie en protestation contre une répression monstrueuse ", Akhbar al-Sharq, en arabe, 27 février 2012.

46. Le Fatah et le Hamas se trouvaient en rupture depuis la reconnaissance manquée de la victoire du Hamas aux élections législatives de 2006 qui avait conduit le mouvement à s'emparer du pouvoir par la force dans la bande de Gaza. 
puis elle prend courage et commence à s'inscrire dans la contestation à travers sa participation aux manifestations dans les rues, d'abord en dehors des camps puis à l'intérieur.

La mobilisation palestinienne montre aussi que cette communauté, en dépit de son ancienneté en Syrie, continue à se projeter dans un horizon d'appartenance différent. C'est pour cette raison que son engagement politique se forge dans une référence double, celle du pays d'accueil et celle du pays d'origine. Des mobilisations proprement locales peuvent alors se charger de multiples sens et cristalliser des attentes qui renvoient à d'autres espaces nationaux où même transnationaux, comme c'est le cas pour la mobilisation qui a vu le jour au cours de la « révolte » syrienne. 